\title{
Endogenous melatonin concentration along with the expression of mitochondrial regulator genes is elevated by the serum shock process in the U87-MG cell line
}

\section{Shayan Balkhi}

Golestan University of Medical Sciences

Marie Saghaeian Jazi

Golestan University of Medical Sciences

Nader Mansour Samaei ( $\nabla$ mansoursamaei@goums.ac.ir )

Golestan University of Medical Sciences

Mahtab Farahmandrad

Golestan University of Medical Sciences

\section{Research Article}

Keywords: Glioblastoma, Melatonin - Cell Proliferation, Cell cycle, Serum shock

Posted Date: March 8th, 2022

DOI: https://doi.org/10.21203/rs.3.rs-881036/v3

License: (c) (1) This work is licensed under a Creative Commons Attribution 4.0 International License.

Read Full License 


\section{Abstract}

According to the World Health Organization, glioblastoma, also known as the fourth grade in the development of astrocytoma, is a glial tumor limited to the central nervous system with a strong ability to invade the brain parenchyma. Melatonin can be generated outside of the pineal gland tissue, according to new research. Melatonin is produced by mitochondria independently but in concert with cell demands, and it plays an important function in cell cycle and metabolism regulation. As a result, we set out to investigate the association between cell metabolism and the serum shock-induced increase in endogenous melatonin, as well as the percentage of cell proliferation.

Background: Melatonin can be produced in the mitochondria organelle of glioblastoma cells without the involvement of the pineal gland, according to new research. Regarding the physiological function of melatonin secreted by the pineal gland in the regulation of rhythmicity, the goal of this study was to see if the glioblastoma cell's melatonin production ability could be influenced using a typical serum shock technique established for cellular rhythm regulator.

Material and methods: First, U87-MG glioblastoma cells were cultured in a DMEM medium containing $10 \%$ FBS and then cells were treated with a standard serum shock process (no FBS, 8h). The concentration of melatonin was measured using ELISA method in supernatant and cell extracts of Shock and control groups. The cell proliferation was measured by using BrdU staining and flow cytometry assessment. The gene expression levels of some mitochondria or circadian related genes including TFAM, BMAL1, PPARGC1A(PGC1-a), and DNM1L(DRP1) were measured, using qRT-PCR method.

Results: In comparison to the control group, serum shock treated U87-MG glioblastoma cells had higher concentrations of cellular and released endogenous melatonin (two times). At the mRNA level, we discovered considerable upregulation of mitochondrial or circadian regulator genes (TFAM, BMAL1, PPARGC1A, and DNM1L); in the shock group compared to the control group $(P<0.0002)$. Furthermore, although the percentage of proliferative cells (Brdu positive) was higher in the shock group, it was not statistically significant.

Conclusion: The serum shock procedure has a significant impact on the U87-MG cell line's cellular activity. In terms of the study's findings, it's worth noting that an increase in endogenous melatonin concentration influences several signaling pathways within the U87-MG cell line, as seen by the increased expression of candidate genes.

In light of the findings of this study, it's worth noting that further research into the role of endogenous melatonin and its effects on cancer cells is critical, and that comparing the results of normal and cancer cells can reveal the hotspots of the signaling pathways involved, which could facilitate in better understanding the biology of glioblastoma.

\section{Introduction}


The most frequent type of primary malignant brain tumor is glioblastoma, which has the greatest fatality rate among patients with primary brain tumors. Although significant progress has been made in understanding the biology of this tumor, it still has a poor prognosis, and additional research is needed to aid in the development of novel therapeutic techniques. Changes in tumour metabolism are critical events in the transformation and adaptation to the microenvironment of the tumor. Glioblastoma cells have previously been shown to use mitochondrial respiration to maintain energy homeostasis under famine conditions. ${ }^{1-2}$ Previous research has focused on the effects of exogenous melatonin injection as a cancer treatment, despite the fact that cancer cells have their own melatonin-based homeostatic system that helps them adjust to alterations. In 1958, melatonin (N-acetyl-5-methoxytryptamine), a hormone released by the pineal gland, was extracted for the first time from a bovine pineal gland. Because the pineal gland of most vertebrates, including humans, secretes this hormone, it is thought to be the primary location of melatonin synthesis. Melatonin, on the other hand, can be produced in the mitochondria of other brain cells. Given that the cell's mitochondria are the primary source of free radicals, produced melatonin can act as a powerful endogenous free radical scavenger to protect the cell from oxidative stress. $^{3-5}$

Melatonin therapy has been shown to reduce the expression of TFAM and other mitochondrial transcription factors including TFB1M and TFB2M, as well as the expression of the mitochondrial NADH dehydrogenase 1 gene in previous research. Changes in the expression of mitochondrial or nuclear genes can result in a decrease in the mitochondrial respiratory chain, which can lead to an increase in ROS generation and apoptosis. ${ }^{6-7}$

The expression of the aryl alkylamine N-acetyltransferase (AANAT) gene is affected by circadian rhythm abnormalities impacting the CLOCK/BMAL1 system. This enzyme is one of the most important components in the manufacture of melatonin and is involved in the conversion of serotonin to melatonin. Melatonin, on the other hand, has been demonstrated to keep a considerable quantity of BMAL1 in cells by inhibiting the proteasome. Melatonin's preventive role is boosted by the discovery of BMAL1 as a tumor suppressor in most cancer cells. ${ }^{8-9}$

It's worth noting that mitochondrial dynamics must be controlled for cellular and tissue bioenergetics to work properly. The DNM1L gene is one of the most significant genes in this pathway. Melatonin's neuroprotective impact is achieved via reduction of oxidative stress and DNM1L-dependent mitochondrial fragmentation, according to studies on Parkinson's model cells. ${ }^{10-11}$

PPARGC1A is one of the most essential mediators of metabolic adaptation. In tandem with enhanced PPARGC1A expression, metabolic and mitochondrial genes have demonstrated increased expression. The TCA cycle, OXPHOS, lipogenesis, and antioxidant genes, for example, were strongly expressed in cells with high PPARGC1A levels. These findings suggest that PPARGC1A gene expression and energy consumption in glioblastoma cells are linked. It also shows that alterations in PPARGC1A expression can play a key role in the Glioblastoma cancer line's proliferation. ${ }^{12-14}$ 
Despite the fact that cancer cells have a melatonin-based homeostatic system that specifically helps them adapt to changes, past research have focused on the effects of exogenous melatonin injection as an effective cancer treatment. These findings, combined with those of previous studies, prompted us to look into one of the melatonin mechanisms in the cell. In order to enhance the concentration of melatonin, circumstances for cellular metabolic stimulation and cellular rhythm readjustment have to be created. $^{4}$

\section{Materials And Methods}

Cell Culture: The human glioblastoma cell line U87-MG (NCBI Code: C531) was purchased from the National Cell Bank of Iran (Pasteur Institute of Iran, Tehran). The cell line was grown in Dulbecco's modified Eagle's medium (DMEM) (GIBCO, USA) and supplemented with $10 \%$ fetal bovine serum (FBS) (GIBCO, USA) and also Penicillin-Streptomycin antibiotic (GIBCO, USA). The cells were then incubated at $37^{\circ} \mathrm{C}$ in a humidified atmosphere of $5 \% \mathrm{CO} 2$ environment.

\section{Serum shock process}

To perform this method, U87-MG cells with an approximate number of $3 \times 105$ cells per well were seeded in 6-well culture plates and placed in a cell culture incubator for 24 hours. when the seeded cells reached to $80 \%$ confluency, the timed process of serum shock was begun.

The number of 3 of 6 existing wells were marked as FBS shock group and the 3 others were considered as the control group; At time $=0$, the medium of shock group was exchanged with serum-rich medium (DMEM + pen strep, supplemented with $50 \%$ horse serum [Bahar Afshan, Iran]), In this 2-hours interval, the cells of the control group were treated with a medium containing $10 \%$ of FBS serum. then, both groups were incubated for 8 hours with serum-free DMEM + pen strep. At the end of the 8-hours period, the cell Supernatant was carefully collected and centrifuged in ribonuclease free micro tubes and placed at minus $70^{\circ} \mathrm{C}$ freezer to be used to measure melatonin. Finally, after the trypsinization process for cell harvesting, cell precipitate was stored in the minus $70^{\circ} \mathrm{C}$ freezer to measure intracellular melatonin, and extract total RNA for studying genes expression.

Melatonin assay with ELISA kit: Melatonin assay was performed on both cell supernatant and cell lysates by (Elabscience Human Melatonin ELISA Kit; lot no: 1RDJVQ8SSG). First Added 50 $\mu \mathrm{L}$ standard or sample to each well. Immediately added $50 \mu \mathrm{L}$ Biotinylated Detection Ab to each well. Micro plate was Incubated for $45 \mathrm{~min}$ at $37^{\circ} \mathrm{C}$. then removed and washed 3 times. after that $100 \mu \mathrm{L}$ HRP Conjugate was Added to each well and incubated for $30 \mathrm{~min}$ at $37^{\circ} \mathrm{C}$. again, Aspirate and wash 5 times. in the next step $90 \mu \mathrm{L}$ Substrate Reagent was Added and Incubate $15 \mathrm{~min}$ at $37^{\circ} \mathrm{C}$. at the end $50 \mu \mathrm{L}$ Stop Solution was added and read at $450 \mathrm{~nm}$ immediately. The concentration of human melatonin in the samples was then determined by comparing the OD of the samples to the standard curve. The results were expressed in $\mathrm{Pg} / \mathrm{mL}$. According the manufacturer the Sensitivity or the minimum detectable dose of Human Melatonin was $9.38 \mathrm{Pg} / \mathrm{mL}$ and the Detection range of method was $15.63-1000 \mathrm{Pg} / \mathrm{mL}$. 
At first, U87-MG cells that had been in the cell culture flask for 24 hours was harvested and seeded to a 6 well cell culture plate. Then the mentioned protocol for serum shock was induced on the cells with a brdu supplementation during the $8 \mathrm{~h}$ incubation time in serum free medium. At the end of 2 hours of shock, all the culture medium was replaced with medium containing $\mathrm{BrdU}(3 \mathrm{mM})$ in a volume of $1 \mathrm{ml}$ of basic culture medium (serum free). After 8 hours and exposure of the cells to BrdU during this time, both cell groups (shock and control groups) were harvested and washed with cold PBS, and centrifuged (At $4{ }^{\circ} \mathrm{C}$ for 3 minutes at $3000 \mathrm{rpm}$ ). And then cells were permeabilized and denaturated using $2 \mathrm{~N} \mathrm{HCL}$ and Triton 100X (0.5) for 30 minutes at room temperature. following 3 times wash with the cold PBS; the cells were stained with anti-BrdU antibody (Monoclonal FITC Mouse IgG1, $\mathrm{k}$ Isotype Ctrl (ICFC) (Bio Legend) with tween20 $(0.5 \%)$ and BSA (1\%) for 90 minutes in the dark. In the last step, the cells were washed 3 times with cold PBS and the proliferation rate was measured by flow cytometry at Blue Laser (488 nm).

\section{Quantitative real-time reverse transcriptase polymerase chain reaction (qRT-PCR):}

Total RNA of the cells was isolated with by RNx Plus extraction kit (SINACLON, Tehran, Iran) and reverse transcribed into cDNA using the cDNA synthesis Kit (YTA, Tehran, Iran). Transcript levels were determined by real-time qRT-PCR using SYBR Green qPCR master mix 2x (100 rxn-Antibody base) (YTA, Tehran, Iran) and performed in a Real-Time PCR Thermal Cycler. The optimal primers for PCR were as follows:

\begin{tabular}{|ll|}
\hline Primer Name & Sequences $\left(5^{\prime} \rightarrow \mathbf{3}^{\prime}\right)$ \\
\hline GAPDH & Forward: 5'- ACAGTCAGCCGCATCTTC - 3' \\
GeneID: 2597 & Reverse: 5'- CTCCGACCTTCACCTTCC - 3 \\
\hline PPARGC1A & Forward: 5'- CCAAAGGATGCGCTCTCGTTCA - 3' \\
GenelD:10891 & Reverse: 5'- CGGTGTCTGTAGTGGCTTGACT - 3' \\
TFAM & Forward: 5'- CCGAGGTGGTTTTCATCTGT - 3' \\
GenelD: 7019 & Reverse: 5'- GCATCTGGGTTCTGAGCTTT - 3' \\
BMAL-1 & Forward: 5'- CAGCCAGTGATGTCTCAAGC - 3' \\
GenelD: 406 & Reverse: 5'- ATGCGTGTCCGTTGTTCC - 3' \\
DNM1L & Forward: 5'- GATGCCATAGTTGAAGTGGTGAC - 3' \\
GenelD: 10059 & Reverse: 5'- CCACAAGCATCAGCAAAGTCTGG - 3' \\
\hline
\end{tabular}

each amplification was performed in triplicate, and expression levels were calculated using the $2-\Delta \Delta C T$ method, with GAPDH serving as the normalization control. 


\section{Results}

\section{Effect of serum shock on U87-MG cell line morphology and proliferation}

The morphology and growth rate of U87-MG cancer cells can be affected by serum shock. Light microscopy was used to observe the morphological changes of the treated cells at first. After 8 hours of serum-free incubation, we observed morphological differences in the shock group, with more elongated oval shaped cells with stretched out cellular extensions, whereas the control group cells had a more circular shape with noticeably fewer extensions (Fig. 1). In comparison to the control group, the 2-hour high serum concentration treatment in the shock group can enhance cells endure the 8-hour serum-free condition. Because the usual serum shock procedure utilized in this work has previously been shown to have a significant impact on cellular metabolic regulation and circadian activation22, the observed morphological alteration could be interpreted in this way.

The proliferative Brdu positive cells were monitored using flowcytometry to investigate the effect of serum shock on the proliferation rate of the U87-MG glioblastoma cancer cells. Our data revealed that the shock-treated group had a higher rate of proliferation, but it was not statistically significant (Fig.2)

\section{Serum shock treatment increase the melatonin concentration in U87-MG glioblastoma cells}

The intracellular and released melatonin concentrations in culture medium were determined using the ELISA method to study the effect of serum shock on melatonin production (content) and release in U87MG glioblastoma cells. With 10+-SE $(\mathrm{Pg} / \mathrm{ml})$ in control vs $35+-\mathrm{SE}(\mathrm{Pg} / \mathrm{ml})$ in shock group $(\mathrm{P}<0.0003)$, we found a substantial increase in melatonin concentration in cell culture supernatant $(P<0.0003)$. (Fig. 3A).

Furthermore, as shown in Fig.3B, the level of measured melatonin in the cell lysate was larger $(\sim>10$ times) than in the supernatant, and the shock group U87-MG cells showed a substantial $(P<0.0003)$ elevated level of melatonin (500+-SE Pg/ml) in contrast to the control (200+-SE Pg/ml).

Serum shock treatment increase the mitochondrial regulator genes expression in U87-MG glioblastoma cells

The gene expression of BMAL-1, one of the primary circadian regulators, was considerably $(P<0.0002)$ elevated, as expected (2+-SE folds increase) serum shock-treated cells, showing that the circadian system has been activated. (See Fig. 4B).

The gene expression experiments showed significant increase of mitochondria transcription factor gene, TFAM (2.5+-SE folds increase, $\mathrm{P}<0.0002$ ) in serum shock group (Fig. 4C). Also, the gene expression of the key mitochondrial fission regulator, DNM1L was upregulated in the serum shock treated U87-MG cells 
in comparison to control, (2+-SE folds increase, $\mathrm{P}<0.0002)$ (Fig. 4D). Moreover, the gene expression of the PPARGC1A transcriptional coactivator that is a central inducer of mitochondrial biogenesis was significantly elevated in serum shock U87-MG cells up to 2 folds of gene expression $(P<0.0002)$ (Fig. 4A). The fact that serum shock can significantly change of the mitochondrion regulator genes including (TFAM, PPARGC1A and DNM1L) indicates potential effect of serum shock in mitochondrial dynamic in response to metabolic effect of serum shock/starvation treatment.

\section{Discussion}

One of the accepted methods for resetting cell rhythm, which regulates cell metabolism and circadian rhythm, is serum shock. ${ }^{17}$. The primary variable in our research was the serum shock process, which was thought to be the fundamental cause of cellular metabolic re - organization. We saw an increase in the rate of proliferation in the shock-group compared to the control group after the serum shock process and exclusion of U87-MG cells from serum-containing media for 8 hours. It's worth noting that the shock group had fewer cellular damages than the control group, implying that morphological differences confirm the difference in proliferation rates between the two groups.

Studies have indicated that Astrocyte cells in the cortex of rats, as well as glioblastoma cells, can produce melatonin independently of pineal cells. ${ }^{15}$ Melatonin produced by a malignant glioma has an antiproliferative endocrine effect; as a result, more aggressive gliomas synthesis or accumulate less melatonin. ${ }^{16}$ We analyze the content of melatonin in cell culture supernatant and cell layset at the same time after serum shock. Melatonin levels in the shock group were two times greater than in the control group. This finding could support the theory that following a serum shock, U87-MG cells begin to release melatonin, which helps them maintain metabolic hemostasis.

The presence of a melatonin transporter in the cell is one of the most recent discoveries in melatonin research. Melatonin is transported by the PEPT1 / 2 membrane transporter in the cell. This protein is found on the cell membrane as well as the mitochondrial membrane, which is the main site of melatonin intercellular production. This transporter's job is to keep melatonin levels in the cell's extracellular and intracellular spaces in check. ${ }^{18}$ The other finding of our investigation was that the levels of melatonin in intercellular and intracellular U87-MG cells differed by about 10-fold. Further research should be conducted to confirm the existence of this transporter in U87-MG cell lines, based on our findings.

The cellular changes that occurred as a result of the serum shock process demanded the evaluation of the expression of specific genes that play key roles in cellular pathways.

The TFAM gene, or mitochondrial transcription factor $A$, was one of the genes investigated. This gene is one of the key controllers of mitochondrial gene transcription and is also important for the mitochondrial genome's preservation and integrity. Injection of exogenous melatonin into the U87-MG cell line lowered the expression of this gene, which enhanced the instability in the mitochondrial genome, resulting in apoptosis of U87-MG cells. ${ }^{6}$ In our work, when endogenous melatonin concentrations were increased following serum shock, the expression of the TFAM gene did not only not drop, but instead increased 
significantly in the shock group compared to the control group. This increase in expression can be attributed to one of the reasons involved in enhancing the rate of proliferation in this study by evaluating the role of TFAM in mitochondrial genome stability.

The PPARGC1A gene is another one that has been investigated. This gene regulates mitochondrial metabolic activity, which is one of the most important aspects of cell metabolism. PPARGC1A affects the action of mitochondrial transcription factors and so plays a role. Increased expression of this gene, on the other hand, boosts mitochondrial biogenesis. Previous research has found that elevated expression of the PPARGC1A gene is related with pathogenesis and malignancy in glioblastoma, particularly in the U87MG cell line. ${ }^{19}$ The expression of the PPARGC1A gene was significantly elevated in our study, which, together with the increase in TFAM expression, could explain why the shock group of the U87-MG cell line was maintained.

The ROR, Rev-erb, and PPARGC1A genes are all promoted by the BMAL1-CLOCK protein complex. Rev-erb, on the other hand, can play a direct role in PPARGC1A expression, mitochondrial biogenesis, and autophagy control. Circadian rhythm changes also influence mitochondrial dynamics by changing DNM1L and ATP levels. ${ }^{20}$ These findings motivated us to examine BMAL1 gene expression during serum shock. The results of the BMAL1 gene expression study revealed that, like the TFAM and PPARGC1A genes, the expression of this gene has increased significantly. This finding implies that serum shock and the consequent increase in endogenous melatonin levels can simultaneously activate many signaling pathways.

Excessive increase or inhibition of PPARGC1A gene expression has been demonstrated to affect biogenesis and mitochondrial dynamics in studies. However, by binding to the promoter of the DNM1L gene and increasing its expression, a moderate increase in the expression of the PPARGC1A gene can directly affect the expression of the DNM1 $\mathrm{L}$ gene, resulting in an increase in mitochondrial dynamics stability. ${ }^{21}$ Following the increase in PPARGC1A gene expression, we decided to evaluate the expression of the DNM1L gene in our investigation. The discovery of a significant increase in DNM1L gene expression demonstrated that the serum shock process leads to the regeneration of cellular metabolic pathways, in which mitochondria play a critical role.

Finally, it can be concluded that after serum shock, U87-MG cells create melatonin, which is accompanied by an increase in the expression of genes that control mitochondrial metabolism and the circadian rhythm. Because of its extensive effect on cellular hemostasis, the involvement of endogenous melatonin in the above pathways should be investigated. Of course, it's worth mentioning that more research into the mechanisms implicated in normal cells and different cancer cell lines is critical.

\section{Declarations}

\section{Acknowledgements}


We would like to thank technical support provided by the Stem Cell Research center and the Human Genetics Department of Golestan University of Medical sciences. Gorgan, Golestan, Iran

\section{Authors' contributions}

S.B carried out the cell experiments, gene expression assays and prepared the manuscript. M.SJ, S.B, and M.F contributed to bioinformatics analysis of data. S. B, M.SJ, and N.MS contributed to project design. All authors read and approved the final manuscript

\section{Funding}

This study was supported by Stem Cell Research Center, Golestan University of Medical Sciences (Grant Number: 31-110455)

\section{Availability of data and materials}

All data generated or analyzed during this study are included in this Manuscript.

\section{Ethics approval and consent to participate}

Not applicable.

\section{Consent for publication}

Informed consent was obtained from all patients

\section{Competing interests}

The authors declare that they have no competing interests

\section{Author details}

1 Department of Human Genetics, Stem Cell Research Center, Golestan University of Medical Sciences

2 Research Assistant Professor of Molecular Medicine, Stem Cell Research Center, Golestan University of Medical Sciences

3* Correspondence: Nader MansourSamaei, Assistant Professor of Genetics, Department of Human Genetics, Stem Cell Research Center, Golestan University of Medical Sciences, Po Box: 4934174611, Gorgan, Iran, Email: mansoursamaei@goums.ac.ir

\section{References}

1. Thakkar, J. P., et al. (2014). "Epidemiologic and Molecular Prognostic Review of Glioblastoma." Cancer Epidemiology Biomarkers \&amp; Prevention: cebp.0275.2014. 
2. Allen, M., et al. (2016). "Origin of the U87MG glioma cell line: Good news and bad news." Science Translational Medicine 8(354): 354re353-354re353.

3. Acuña-Castroviejo, D., Escames, G., Venegas, C. et al. Extrapineal melatonin: sources, regulation, and potential functions. Cell. Mol. Life Sci. (2014) 71: 2997.

4. Suofu, Y., et al. (2017). "Dual role of mitochondria in producing melatonin and driving GPCR signaling to block cytochrome c release." Proceedings of the National Academy of Sciences 114(38): E7997-E8006.

5. Bondy, S. C. and A. Campbell (2018). "Mechanisms Underlying Tumor Suppressive Properties of Melatonin." International Journal of Molecular Sciences 19(8): 2205.

6. Lezza, A. M. S. (2012). "Mitochondrial transcription factor A (TFAM): one actor for different roles." Frontiers in Biology 7(1): 30-39.

7. Lee, H., Park, J., Tran, Q., Kim, D., Hong, Y., Cho, H. ... Park, J. (2017). Mitochondrial transcription factors A (TFAM) is upregulated in glioma. Molecular Medicine Reports, 15, 3781-3786.

8. Khan S, Liu Y, Siddique R, Nabi G, Xue M, Hou H. Impact of chronically alternating light-dark cycles on circadian clock mediated expression of cancer (glioma)-related genes in the brain. Int J Biol Sci 2019; 15(9):1816-1834.

9. Chuffa, L., Seiva, F., Cucielo, M., Silveira, H., Reiter, R.J. and Lupi, L.A. 2019. Clock genes and the role of melatonin in cancer cells: an overview. Melatonin Research. 2, 2 (Jun. 2019), 133-157.

10. Schmitt, K., et al. (2018). "Circadian Control of DRP1 Activity Regulates Mitochondrial Dynamics and Bioenergetics." Cell Metabolism 27(3): 657-666.e65

11. Chuang, J.-I., et al. (2016). "Melatonin prevents the dynamin-related protein 1-dependent mitochondrial fission and oxidative insult in the cortical neurons after 1-methyl-4-phenylpyridinium treatment." Journal of Pineal Research 61(2): 230-240

12. Cho, S.Y., Kim, S., Yi, M., Zhang, E., Kim, E., Park, J. ... Kim, D.W. (2017). Expression of PGC1a in glioblastoma multiforme patients. Oncology Letters, 13, 4055-4076.

13. Bruns, l., et al. (2018). "Disruption of Peroxisome proliferator-activated receptor coactivator 1-alpha (PGC-1a) reverts key features of the neoplastic phenotype of glioma cells." Journal of Biological Chemistry

14. Martín, V., et al. (2006). "Intracellular Signaling Pathways Involved in the Cell Growth Inhibition of Glioma Cells by Melatonin." Cancer Research 66(2): 1081-1088

15. Neamati, F. and Asemi, Z. (2020), The effects of melatonin on signaling pathways and molecules involved in glioma. Fundam Clin Pharmacol, 34: 192-199. doi.org/10.1111/fcp.12526

16. Kinker, G.S., Oba-Shinjo, S.M., Carvalho-Sousa, C.E., Muxel, S.M., Marie, S.K.N., Markus, R.P. and Fernandes, P.A. (2016), Melatonergic system-based two-gene index is prognostic in human gliomas. J. Pineal Res., 60: 84-94.doi.org/10.1111/jpi.12293

17. Xiang S, Mao L, Duplessis T, et al. Oscillation of Clock and Clock Controlled Genes Induced by Serum Shock in Human Breast Epithelial and Breast Cancer Cells: Regulation by Melatonin. Breast Cancer: 
Basic and Clinical Research. January 2012. doi:10.4137/BCBCR.S9673

18. Reiter, R. J., et al. (2018). "Mitochondria: Central Organelles for Melatonin's Antioxidant and AntiAging Actions." Molecules 23(2): 509.

19. Ferese, R., et al. (2020). "Quantitative Ultrastructural Morphometry and Gene Expression of mTORRelated Mitochondriogenesis within Glioblastoma Cells." International Journal of Molecular Sciences 21(13): 4570.

20. Zhang, H., et al. (2020). "Do not neglect the role of circadian rhythm in muscle atrophy." Ageing Research Reviews 63: 101155.

21. Dabrowska, Aleksandra et al. "PGC-1 a controls mitochondrial biogenesis and dynamics in leadinduced neurotoxicity." Aging vol. 7,9 (2015): 629-47. doi:10.18632/aging.100790

22. Izumo, M., et al. (2006). "Quantitative Analyses of Circadian Gene Expression in Mammalian Cell Cultures." PLOS Computational Biology 2(10): e136.

\section{Figures}
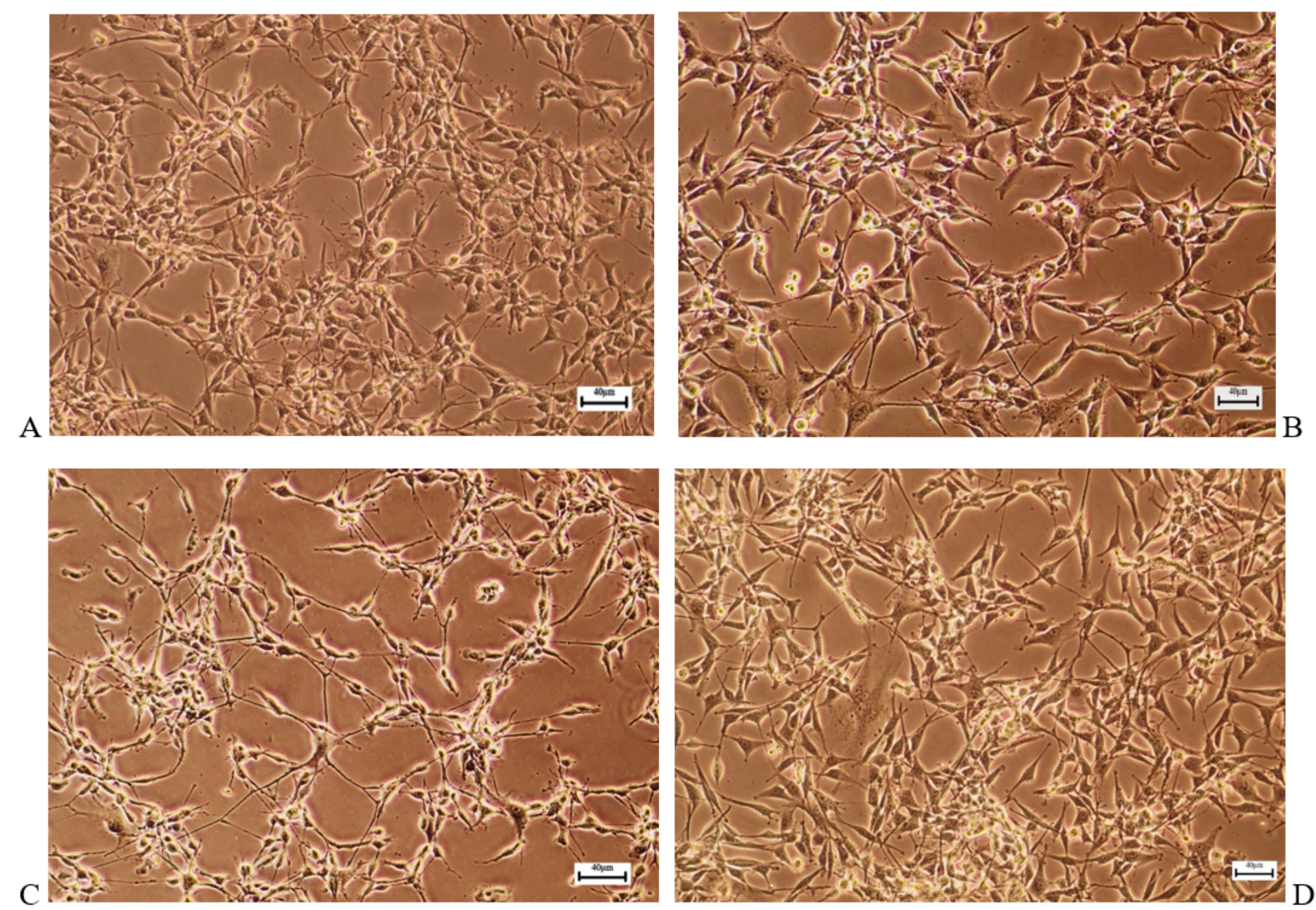

Figure 1 
morphological changes of U87-MG glioblastoma cell line in response to serum shock. A) Control group after $2 \mathrm{~h}$ shcok process with $10 \%$ FBS, B) Shock group after $2 \mathrm{~h}$ shock process with $50 \%$ Horse serum, C) Control Group after $8 \mathrm{~h}$ in serum free medium, D) shock Group after $8 \mathrm{~h}$ in serum free medium

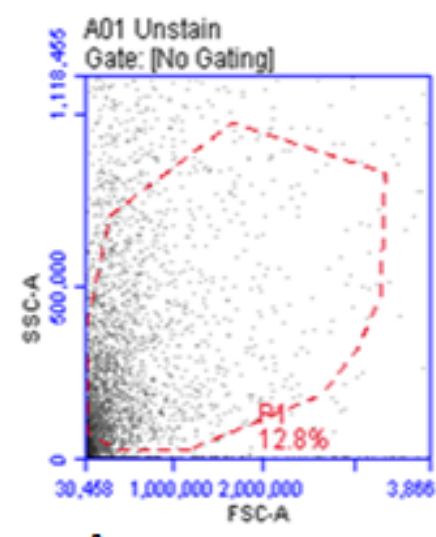

A

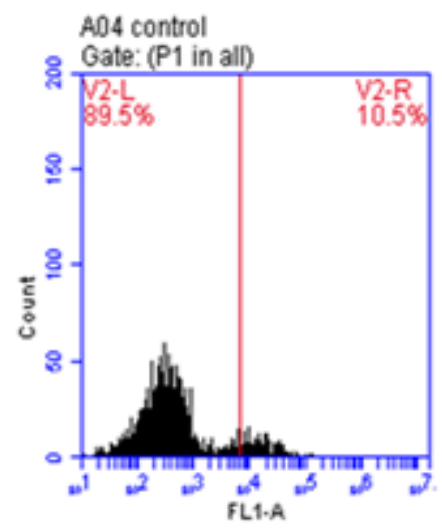

D

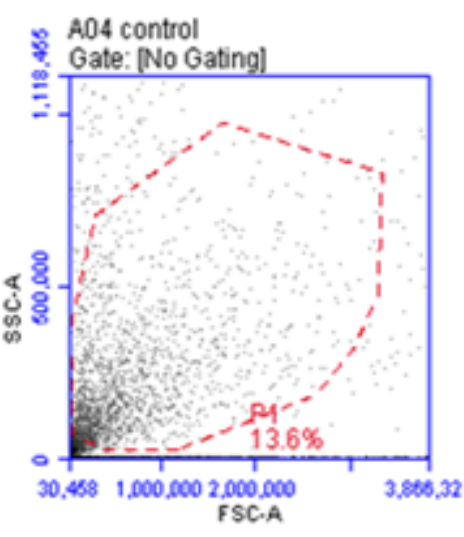

B

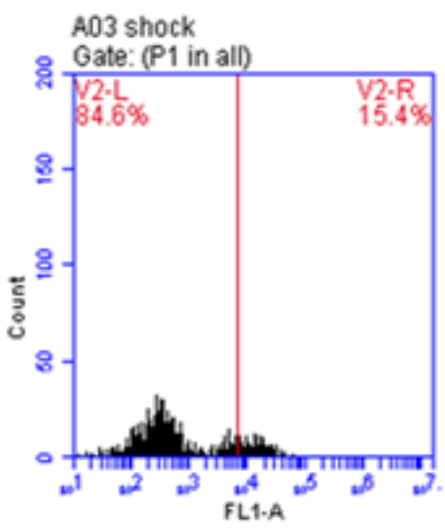

E

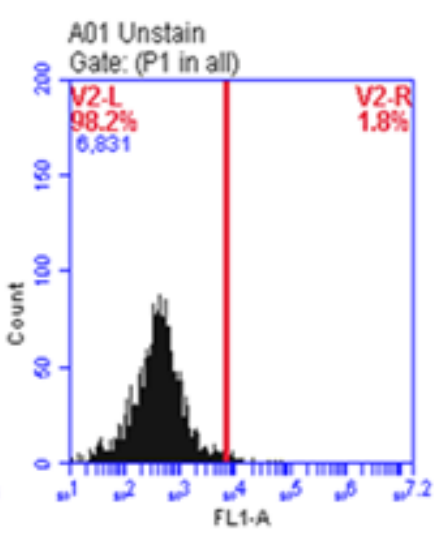

C

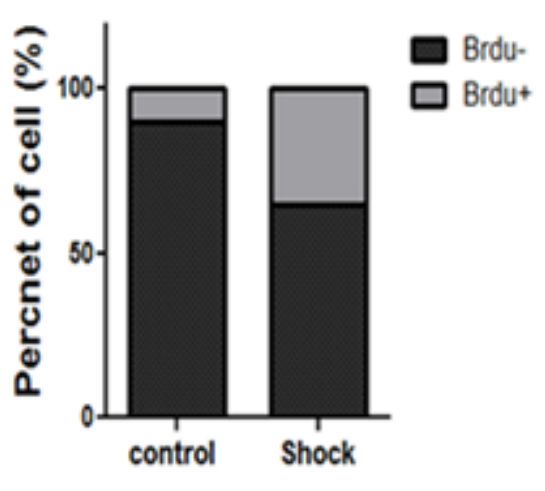

Figure 2

Evaluation of U87-MG cellular proliferation rate by BrdU staining method. The A and B show the forward to side scatter plot of un-stain (A) and control (B) U87-MG cells. The sample histogram plots are shown for un-stain (C), control (D) and serum shock (E) treated U87-MG cells, which Fl-1 indicates BrdU positive cells. The bar chart shows mean of BrdU+ and BrdU- U87-MG cells in each group. 

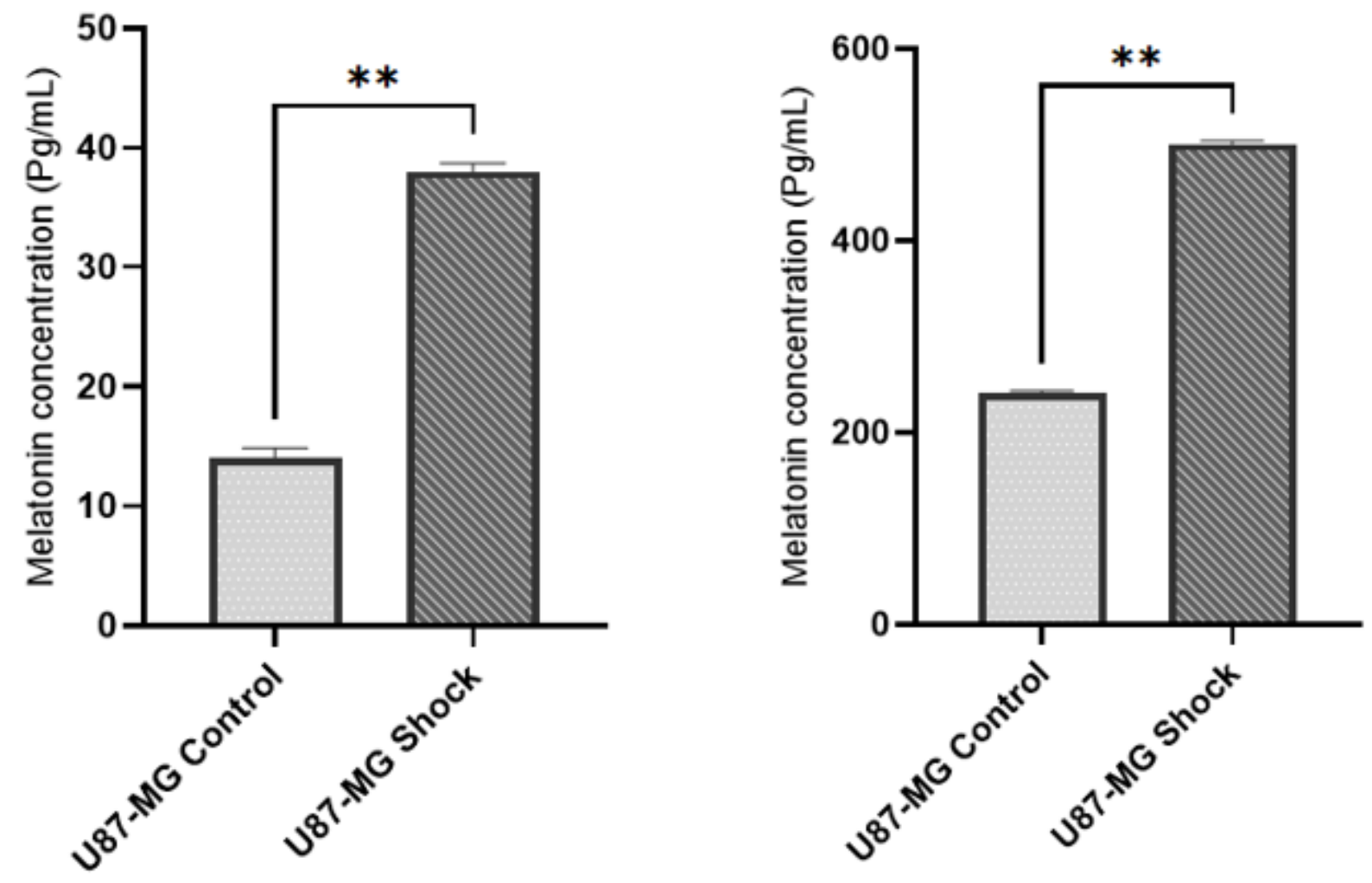

A

B

Figure 3

Level of the released (A) and intracellular (B) melatonin concentration in U87-MG cells in response to serum shock. 

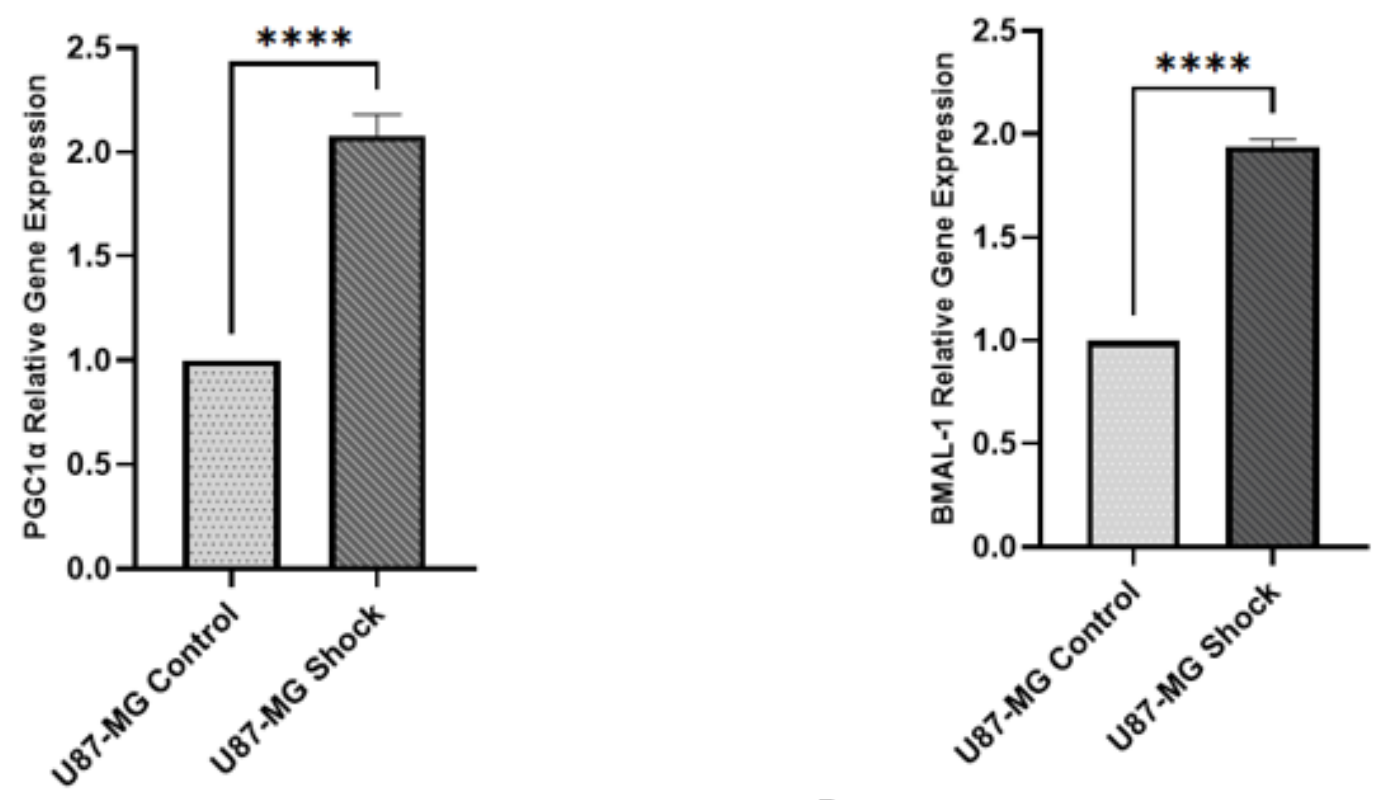

A

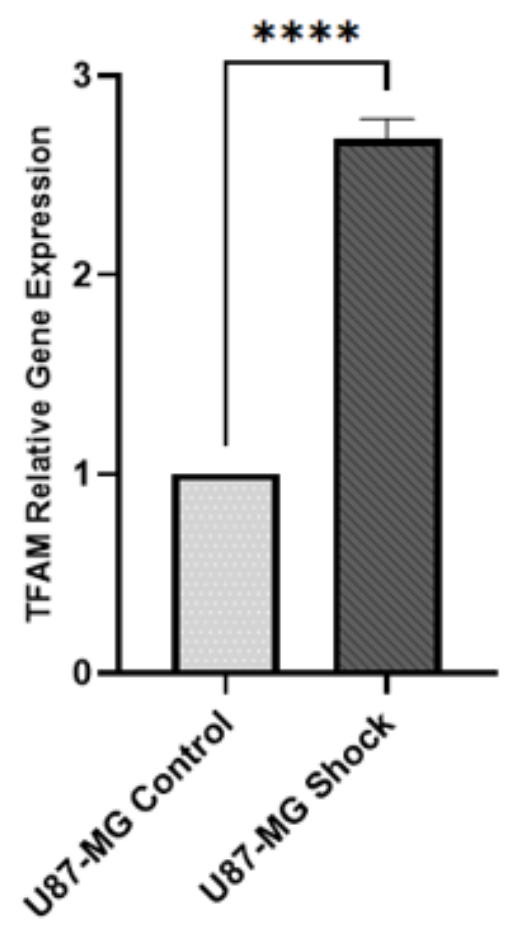

C

B

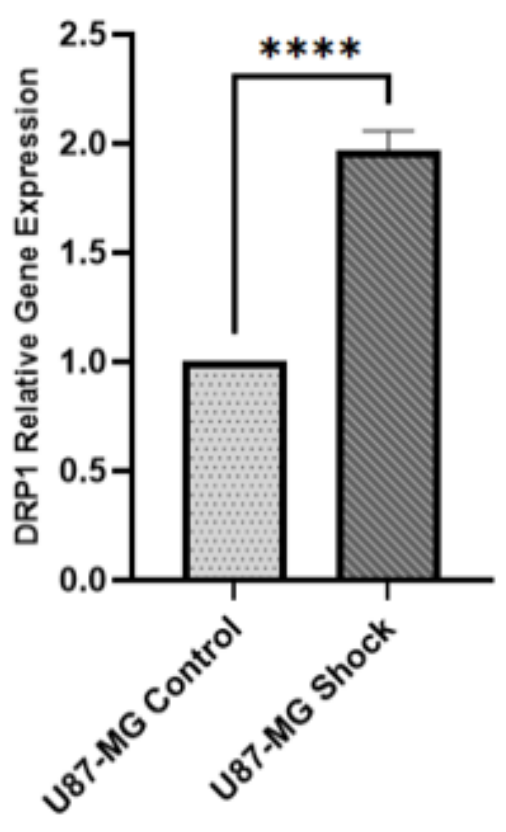

D

Figure 4

Gene Expressions: Each amplification was performed in triplicate, and expression levels were calculated using the 2- $\triangle \triangle C T$ method, with GAPDH serving as the normalization control. A) the expression of PPARGC1A (PGC1a) gene in U87-MG shock group and control group. B) the expression of BMAL1 gene in U87-MG shock group and control group. C) the expression of TFAM gene in U87-MG shock group and control group. D) the expression of DNM1L (DRP1) gene in U87-MG shock group and control group. 
Page 15/15 